\title{
Lysosomotropic agents including azithromycin, chloroquine and hydroxychloroquine activate the integrated stress response
}

\author{
Ai-Ling Tian ${ }^{1,2}$, Qi Wu ${ }^{1,2,3}$, Peng Liu (10 ${ }^{1,2}$, Liwei Zhao ${ }^{1,2}$, Isabelle Martins ${ }^{1,2}$, Oliver Kepp $\mathbb{B}^{1,2}$, Marion Leduc ${ }^{1,2}$ and \\ Guido Kroemer ${ }^{1,2,5,6}$
}

\begin{abstract}
The integrated stress response manifests with the phosphorylation of eukaryotic initiation factor $2 a$ (elF2a) on serine residue 51 and plays a major role in the adaptation of cells to endoplasmic reticulum stress in the initiation of autophagy and in the ignition of immune responses. Here, we report that lysosomotropic agents, including azithromycin, chloroquine, and hydroxychloroquine, can trigger elF2a phosphorylation in vitro (in cultured human cells) and, as validated for hydroxychloroquine, in vivo (in mice). Cells bearing a non-phosphorylatable elF2a mutant $(\mathrm{S} 51 \mathrm{~A})$ failed to accumulate autophagic puncta in response to azithromycin, chloroquine, and hydroxychloroquine. Conversely, two inhibitors of elF2a dephosphorylation, nelfinavir and salubrinal, enhanced the induction of such autophagic puncta. Altogether, these results point to the unexpected capacity of azithromycin, chloroquine, and hydroxychloroquine to elicit the integrated stress response.
\end{abstract}

\section{Introduction}

Azithromycin (AZT), chloroquine (CQ), and 3hydroxychloroquine (HCQ) have attracted much attention over the past months as possible (and controversial) therapeutic agents for the treatment of coronavirus disease-19 (COVID-19) ${ }^{1,2}$. At this point, it has not been resolved whether the frequently administered combination regimen of AZT and HCQ (often supplemented with zinc) itself reduces the morbidity and mortality of COVID-19 or whether accompanying measures (such as provision of anti-diabetic, anti-hypertensive, anti-inflammatory, and/or anti-thrombotic agents) or even placebo effects account for the clinical efficiency of AZT $+\mathrm{HCQ}$,

\footnotetext{
Correspondence: Oliver Kepp (captain.olsen@gmail.com) or Guido Kroemer (kroemer@orange.fr)

${ }^{1}$ Centre de Recherche des Cordeliers, Equipe labellisée par la Ligue contre le cancer, Université de Paris, Sorbonne Université, Inserm U1138, Institut Universitaire de France, Paris, France

${ }^{2}$ Metabolomics and Cell Biology Platforms, Gustave Roussy, Villejuif, France Full list of author information is available at the end of the article These authors contributed equally: Ai-Ling Tian, Qi Wu, Marion Leduc Edited by G. Melino
}

which are more frequently observed in retrospective analyses and uncontrolled clinical studies ${ }^{3-5}$ than in prospective randomized studies ${ }^{6-9}$.

AZT is a macrolide antibiotic, while CQ and HCQ are antimalarial drugs. HCQ is also been widely used for the treatment of rheumatoid arthritis and systemic lupus erythematosus ${ }^{10,11}$. All the three agents are lysosomotropic ${ }^{12-14}$, meaning that they are sufficiently lipophilic to penetrate into cells but also weak bases so that they get protonated at low $\mathrm{pH}$ to become trapped in lysosomes, hence gradually increasing their concentration in the lysosomal lumen until they destabilize lysosomal membranes due to detergent-like effects, causing a loss of lysosomal acidification and blockade of lysosomal functions ${ }^{15,16}$ that ultimately activates homeostatic circuitries including the activation of transcription factors such as TFEB and TFE3 for lysosomal biogenesis ${ }^{17}$. In addition, the loss of lysosomal acidity/function observed in cells treated with AZT, CQ, or HCQ results in the blockade of lysosomal fusion with autophagosomes, thus stalling autophagic flux and causing the accumulation of autophagosomes that cannot be eliminated ${ }^{18-20}$. Moreover, CQ and

\section{(c) The Author(s) 2021}

(c) (i) Open Access This article is licensed under a Creative Commons Attribution 4.0 International License, which permits use, sharing, adaptation, distribution and reproduction c. in any medium or format, as long as you give appropriate credit to the original author(s) and the source, provide a link to the Creative Commons license, and indicate if changes were made. The images or other third party material in this article are included in the article's Creative Commons license, unless indicated otherwise in a credit line to the material. If material is not included in the article's Creative Commons license and your intended use is not permitted by statutory regulation or exceeds the permitted use, you will need to obtain permission directly from the copyright holder. To view a copy of this license, visit http://creativecommons.org/licenses/by/4.0/. 
HCQ can stimulate lysosomal membrane permeabilization that secondarily elicits the mitochondrial pathway of apoptosis $^{21}$, hence resulting in cell death, likely contributing to the toxicity of these agents ${ }^{22,23}$.

The integrated stress response (ISR) consists in the phosphorylation of the phylogenetically conserved eukaryotic initiation factor $2 \alpha$ (eIF $2 \alpha)$ by a series of eIF $2 \alpha$ kinases (EIF2K1 to 4) and plays a cardinal role in the adaptation of stress to endoplasmic reticulum (ER) stress (in particular, the accumulation of unfolded or misfolded proteins in the ER lumen) ${ }^{24}$, in the innate cellular defense against viral infections (to block the translation of virusencoded RNAs into protein $)^{25-27}$, as well as in the initiation of autophagy (which also can lead to the elimination of intracellular pathogens) $)^{28-31}$. Moreover, eIF2 $\alpha$ phosphorylation contributes to the phenomenon of "immunogenic cell death" (ICD) ${ }^{32-34}$, which likely plays a major role in connecting the virus-induced death of infected cells to immune response that ultimately lead to the active elimination of virus-infected cells by cytotoxic $\mathrm{T}$ lymphocytes ${ }^{35-37}$. This latter effect is achieved due to the contribution of eIF $2 \alpha$ phosphorylation to (i) autophagy, which enables the lysosomal secretion of ATP (which is a major chemoattractant for dendritic cell precursors) ${ }^{28,29,31,38}$ and (ii) the exposure of the ER lumen protein calreticulin at the cell surface (where it acts as an eat-me signal to render dying/dead cells palatable to dendritic cells, allowing them to present viral antigens to $\mathrm{T}$ lymphocytes) ${ }^{33,39-41}$.

In view of the considerable (patho)physiological relevance of ISR, we decided to investigate whether AZT, CQ, or HCQ may induce this phenomenon. Here, we show that these three agents induce signs of ISR in vivo, and that ISR contributes to the accumulation of stalled autophagosomes as well as to the cytotoxicity of these agents.

\section{Results}

\section{Lysosomotropic agents induce elF2a phosphorylation}

\section{in vitro}

Human U2OS osteosarcoma cells stably expressing a GFP-LC3 fusion protein exhibit GFP-LC3 dots in the cytoplasm (corresponding to "autophagic puncta") ${ }^{42}$ in response to the autophagy inducer torin1 (TOR, an inhibitor of mechanistic target of rapamycin, mTOR) and the lysosomal inhibitor bafilomycin A1 (BafA1, an inhibitor of the vacuolar-type $\mathrm{H}^{+}$-ATPase (V-ATPase) that is required for lysosomal acidification $)^{43}$. Similar to BafA1, the three lysosomotropic agents AZT, CQ, and HCQ did not cause any cytotoxicity in the timeframe of the experiment (Fig. 1A-D) but stimulated a dosedependent increase in GFP-LC3 dots. The formation of GFP-LC3 puncta was observed in wild-type U2OS and human glioma $\mathrm{H} 4$ cells but not in cells that are deficient for the essential autophagy protein ATG5 and which acts upstream of LC3 to facilitate lipidation and membrane association (Fig. 1E, F and Supplementary Fig. 1). Moreover, AZT, CQ, and HCQ stimulated the translocation of the transcription factors TFEB and TFE3 from the cytoplasm to the nuclei, as determined in U2OS cells expressing a GFP-TFEB fusion protein (Fig. 1G, H) or by immunofluorescence detection of TFE3 (Fig. 1I, J). AZT, $\mathrm{CQ}$, and HCQ inhibited autophagic flux in U2OS RFPGFP-LC3 tandem reporter cells, as can be expected from agents that perturb lysosomal function (Supplementary Fig. 2) $)^{15,16,44}$. In addition, AZT, CQ, and HCQ induced the phosphorylation of eIF2 $\alpha$ (as measured by immunofluorescence and immunoblot using a phosphoneoepitope-specific antibody) (Fig. 2A, B and Supplementary Fig. 3$)^{45}$, the activation of the transcription factor CHOP (as indicated by the expression of GFP placed under the control of the CHOP promoter) (Fig. 2C, D), the upregulation of ATF6 (as indicated by the expression of an ATF6-GFP fusion protein) (Fig. 2E, $\mathrm{F}$ ), and the activation of XBP1 (as indicated by the expression of an XBP1-GFP/Venus fusion protein in which GFP/Venus is only expressed after that IRE1 $\alpha$ has caused the splicing of the corresponding mRNA (Fig. $2 \mathrm{G}, \mathrm{H})$. However, in quantitative terms, the effects of AZT, CQ, and HCQ on CHOP, ATF6, and XBP1 appear relatively minor when compared to the positive controls thapsigargin and tunicamycin employed to elicit ER stress (Fig. $2 \mathrm{C}-\mathrm{H}$ ). Only the level of eIF $2 \alpha$ phosphorylation induced by AZT, CQ, and HCQ reaches that of the positive controls (Fig. 2A, B). Similarly, CQ and HCQ (but not AZT) induced a relatively low level of NF$\mathrm{kB}$ activation as compared to the positive control, tumor necrosis factor- $\alpha$ (Supplementary Fig. 4). We conclude that AZT, CQ, and HCQ are potent perturbators of lysosomal function as well as potent inducers of the ISR consisting in eIF $2 \alpha$ phosphorylation.

\section{elF2a phosphorylation is required for the induction of autophagic puncta}

TFEB and TFE3 are well known pro-autophagic transcription factors ${ }^{46,47}$. Accordingly, their double knockout (DKO) attenuated the induction of GFP-LC3 puncta by AZT, CQ, and HCQ (Fig. 3A, B). Many autophagy inducers require eIF $2 \alpha$ phosphorylation as a mandatory step for the ignition of the process ${ }^{48}$. Accordingly, we observed that a knockin mutation that renders eIF $2 \alpha$ non-phosphorylatable (due to the replacement of serine in position 51 by an alanine residue: genotype $e I F 2 \alpha^{551 \mathrm{~A} / \mathrm{S} 51 \mathrm{~A}}$ ) strongly inhibited the induction of GFP-LC3 puncta by AZT, CQ, and HCQ (Fig. 3C, D). Similarly, inhibition of ER stress with the chemical chaperone 4-phenylbutyric acid (4-PBA) ${ }^{49}$ attenuated the induction of GFP-LC3 puncta by AZT, CQ, and HCQ (Fig. 4A, B). Conversely, treatment of the cells with two inhibitors of eIF2 $\alpha$ dephosphorylation, nelfinavir ${ }^{50}$ and 


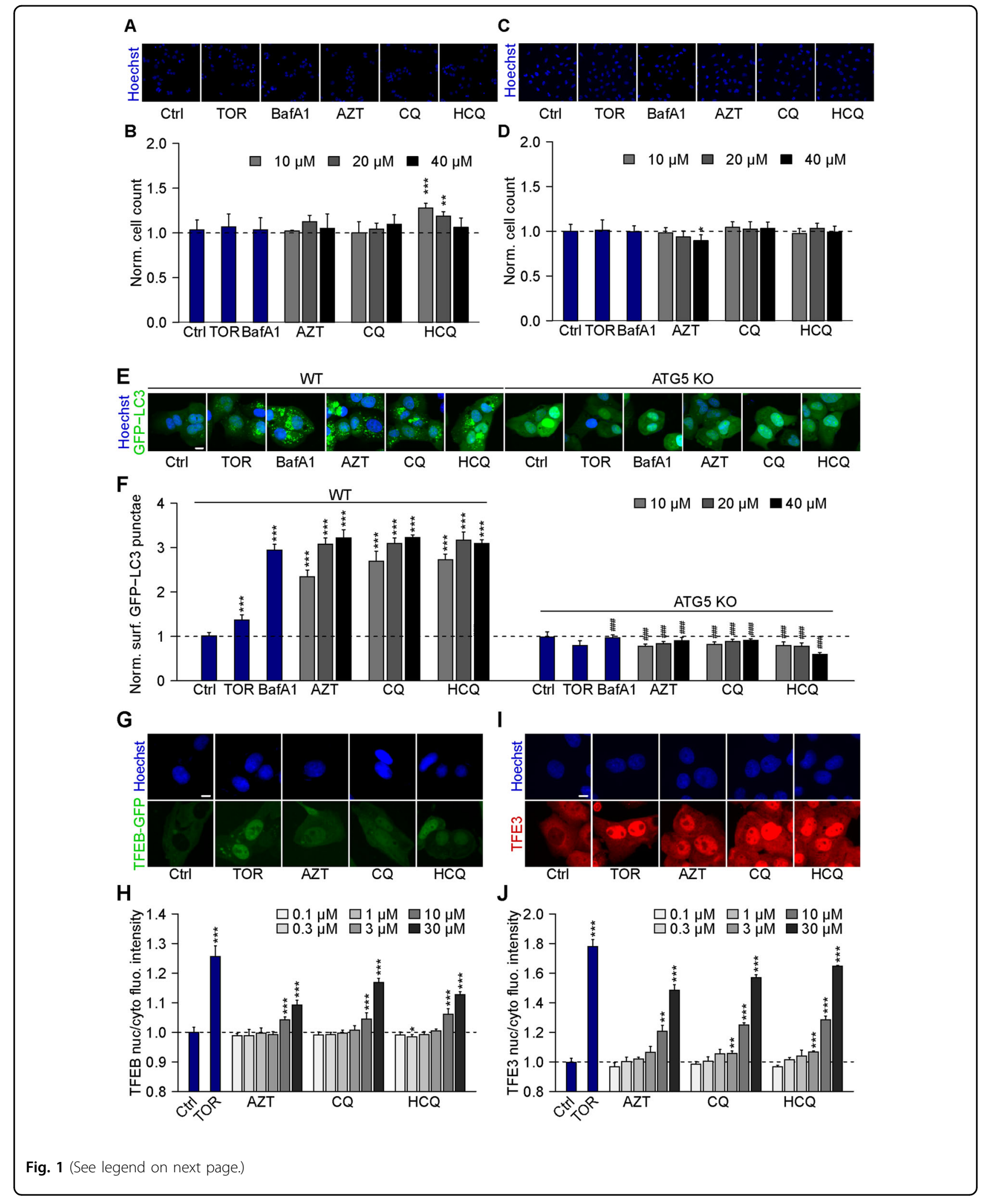




\begin{abstract}
(see figure on previous page)
Fig. 1 Chloroquine, hydroxychloroquine, and azithromycin induce the formation of LC3 puncta and trigger TFEB/TFE3 translocation. A-D Human osteosarcoma U2OS-GFP-LC3 (A, B) or human glioma H4-GFP-LC3 cells (C, D) were treated with chloroquine (CQ; 10, 20, 40 $\mu$ M), hydroxychloroquine (HCQ; 10, 20,40 $\mu \mathrm{M}$ ), azithromycin (AZT; 10, 20, $40 \mu \mathrm{M})$, the autophagy inducer torin 1 (TOR; $300 \mathrm{nM}$ ), or the inhibitor of autophagic flux bafilomycin A1 (BafA1; $100 \mathrm{nM}$ ) for $6 \mathrm{~h}$. After fixation, healthy cells depicted by normal nuclear morphology were enumerated. Representative microscopical images are shown in $\mathbf{A}$ and $\mathbf{C}(A Z T, C Q$, and $H C Q, 40 \mu M)$ and normalized mean data are depicted as bar charts in $\mathbf{B}$ and D. Data are means \pm SD of four replicates $\left({ }^{*} P<0.05,{ }^{* *} P<0.01,{ }^{* * *} P<0.001\right.$ vs. vehicle control (Ctrl); Student's $t$-test). E, F U2OS-GFP-LC3 wild type or

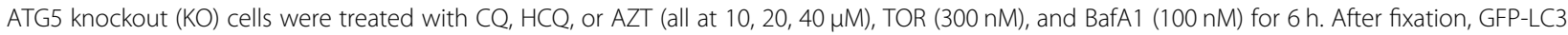
dots were analyzed as a proxy for autophagy induction. Representative microscopical images are shown in $\mathbf{E}(\mathrm{AZT}, \mathrm{CQ}$, and $\mathrm{HCQ}, 40 \mu \mathrm{M})$ and normalized mean data are depicted as bar chart in $\mathbf{F}$. Data are means \pm SD of four replicates ${ }^{* *} P<0.01,{ }^{* *} P<0.001$ vs. vehicle control (Ctrl), and ${ }^{\# \# \# P}$ $<0.001$ vs. WT; Tukey's multiple comparisons test). G, H U2OS cells stably expressing GFP-TFEB fusion protein were treated with CQ, HCQ, or AZT (all at $0.1,0.3,1,3,10,30 \mu \mathrm{M})$ for $6 \mathrm{~h}$. TOR at $300 \mathrm{nM}$ was used as a positive control for TFEB nuclear translocation. Images were analyzed and the ratio of GFP intensities in nuclei and cytoplasm was calculated to indicate TFEB translocation to nuclei $(\mathbf{H})$. Representative images are depicted in $\mathbf{G}(A Z T, C Q$, and HCQ, $30 \mu \mathrm{M}$ ). I, J U2OS cells were treated as above, and then TFE3 translocation was assessed microscopically after immunostaining (I). TOR at $300 \mathrm{nM}$ was used as a positive control for TFE3 nuclear translocation. TFE3 intensities in the nucleus and the cytoplasm were measured, and the nucleo-to-cytoplasmic ratio of TFE3 intensities was calculated to indicate nuclear translocation of TFE3 $(\mathbf{J})$. Data are means \pm SD of four replicates $\left({ }^{*} P\right.$ $<0.05,{ }^{* *} P<0.01,{ }^{* *} P<0.001$ vs. Ctrl, Student's $t$-test). Scale bars equal $10 \mu \mathrm{m}$.
\end{abstract}

salubrinal ${ }^{51}$, enhanced the formation of GFP-LC3 puncta in response to AZT, CQ, and HCQ (Fig. 4A, B and Supplementary Fig. 5).

In accord with previous work ${ }^{21}$, CQ and HCQ induces some degree of cellular toxicity, leading to the manifestation of apoptotic and necrotic events that can be distinguished by dual staining with annexin-V-FITC (which stains apoptotic and necrotic cells) the vital dye 4',6-diamidino-2-phenylindole (DAPI, which only stains necrotic cells) ${ }^{52}$. Among the genotypes evaluated in this paper $\left(A_{T G 5}{ }^{-1-}\right.$, eIF $2 \alpha^{\text {S51A/S51A }}, T_{F E B}{ }^{-1-}, T_{F E 3}{ }^{-1-}$, TFEB/ TFE3 DKO, PERK ${ }^{-1-}$ ) the $e I F 2 \alpha^{\mathrm{S} 51 \mathrm{~A}}$ knockin mutation rendering eIF2 $\alpha$ non-phosphorylatable had the strongest effect on apoptosis induction by CQ and HCQ (Fig. 5A), increasing cellular killing by CQ and HCQ but not by the general tyrosine kinase inhibitor and apoptosis inducer staurosporin (STS) (Fig. 5B and Supplementary Fig. 6). These results point to the ISR as central for the effects of $\mathrm{CQ}$ and $\mathrm{HCQ}$.

\section{Lysosomotropic agents induce elF2a phosphorylation in vivo}

The aforementioned results have been obtained in vitro, calling for their in vivo validation. For this, we injected mice intraperitoneally with HCQ (at a dose that inhibits autophagic flux $)^{53-56}$ alone or in combination with AZT (supplemented in the drinking water). Of note, HCQ (but less so AZT) induced a remarkable and significant increase in eIF2 $\alpha$ phosphorylation that was detectable by immunoblot in liver extracts (Fig. 6A, B) but less so in the myocardium (Supplementary Fig 7). In addition, one single injection of HCQ was able to stimulate a significant increase in eIF2 $\alpha$ phosphorylation in several circulating leukocyte subsets (in particular neutrophil granulocytes, lymphocytes, and monocytes), as determined by immunofluorescence staining and imaging flow cytometry (Fig. 6D, E). Thus, HCQ can induce eIF2 $\alpha$ phosphorylation in vivo, supporting the capacity of this agent to activate ISR.

\section{Discussion}

As we show in this work, lysosomotropic agents including $\mathrm{AZR}, \mathrm{CQ}$, and $\mathrm{HCQ}$ are capable of stimulating the ISR. The capacity of these agents to induce the cardinal hallmark of ISR, eIF2 $\alpha$ phosphorylation, is observed at similar concentrations as those required to induce the accumulation of autophagic puncta and to activate the transcription factors TFEB and TFE3 in a dose of $10-40 \mu \mathrm{M}$. The accumulation of autophagic puncta induced by AZT, CQ, and HCQ requires the initial steps of autophagy, as illustrated by the fact that ATG5deficient cells fail to demonstrate this phenomenon. This is in accordance with findings showing that CQ can induce non-canonical V-ATPase-dependent LC3 lipidation $^{57}$. Moreover, AZT, CQ, and HCQ were unable to elicit the accumulation of LC3-binding autophagosomes in cells expressing a non-phosphorylatable mutant of eIF2 $\alpha$, suggesting causality between ISR and the observed phenomenon. This conjecture was further supported by the observation that two inhibitors of the dephosphorylation of eIF2 $\alpha$ enhanced autophagosome accumulation in vitro. Moreover, the apoptosis-inducing effect of CQ and HCQ was reduced in cells bearing mutant eIF $2 \alpha$.

The ISR plays a major role in the inhibition of viral replication. Indeed, multiple viruses have developed strategies to subvert the ISR, either by directly inhibiting eIF $2 \alpha$ kinases or by deploying a decoy that resembles eIF $2 \alpha$, hence preventing the phosphorylation of the cellular protein ${ }^{25,58,59}$. In addition, a protein encoded by coronavirus counteracts the ISR at its very core by acting as a competitive inhibitor of the phospho-eIF $2 \alpha$-eIF $2 \beta$ 


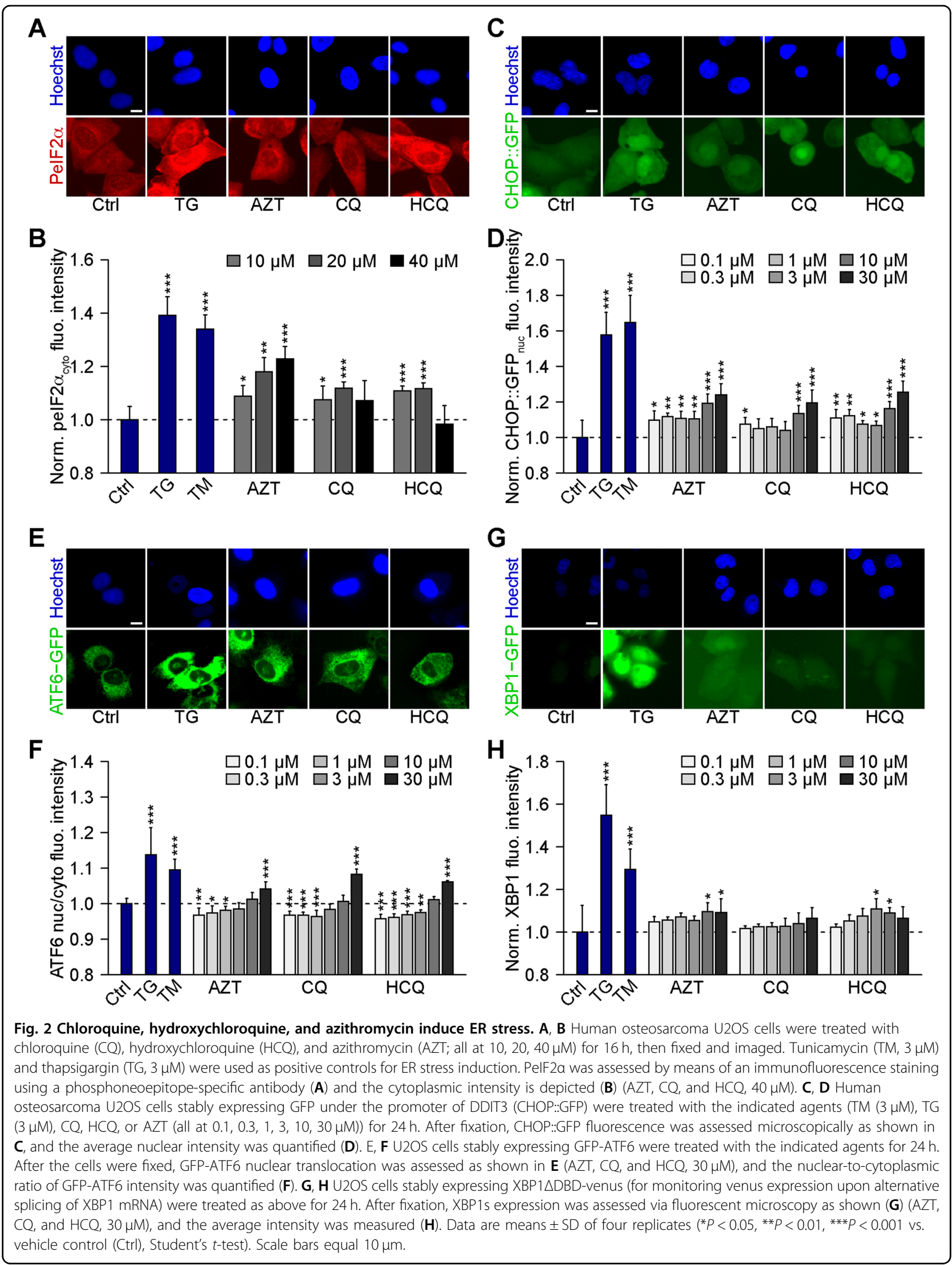



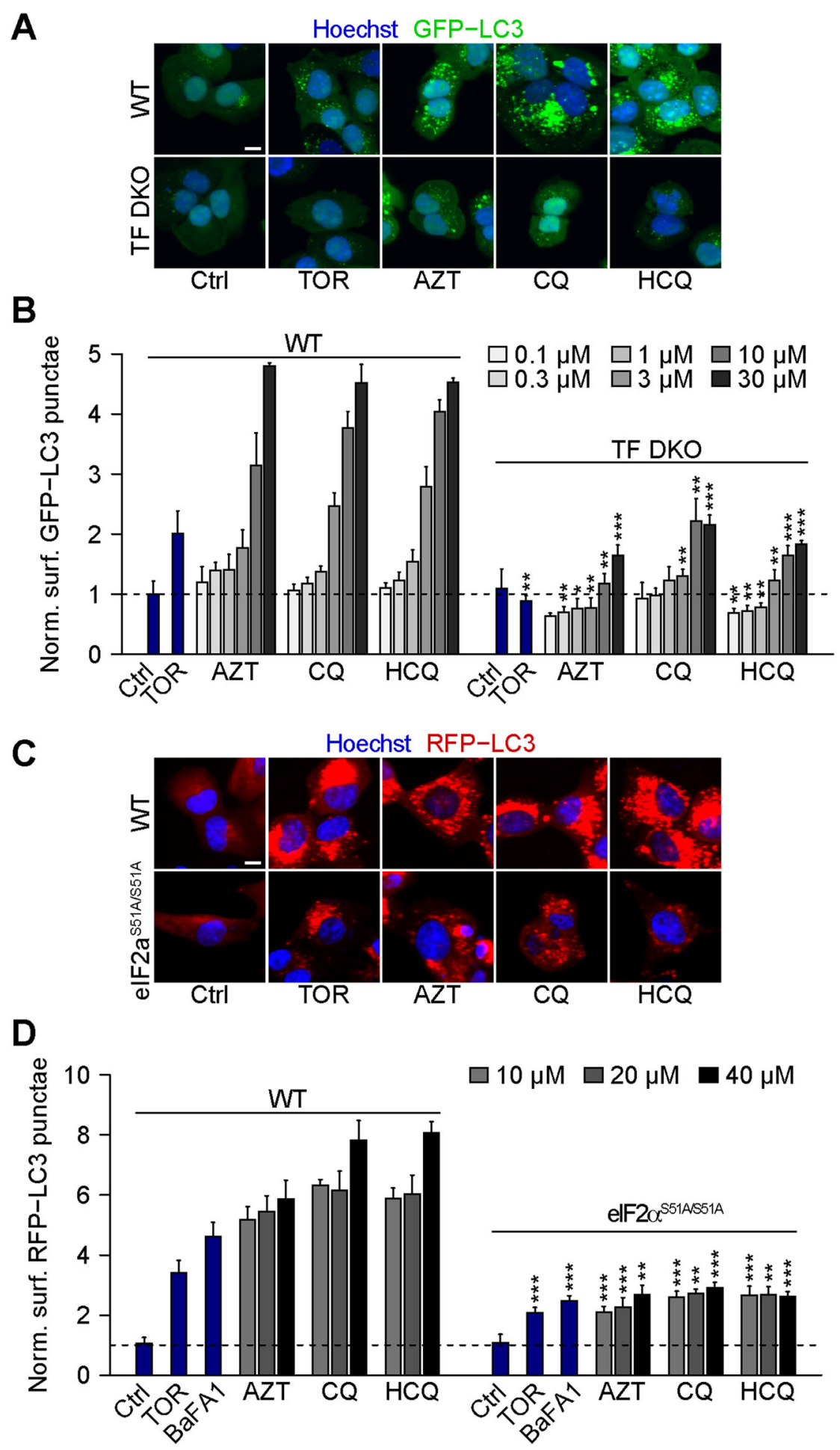

Fig. 3 Chloroquine, hydroxychloroquine, and azithromycin-induced autophagy depends on TFEB/TFE3 and elF2a. A, B Human

osteosarcoma U2OS wild type (WT) or TFEB/TFE3 double KO (TF DKO) cells both stably expressing GFP-LC3 were treated with the indicated compounds (torin 1 (TOR; $300 \mathrm{nM}$ ), chloroquine (CQ), hydroxychloroquine (HCQ), and azithromycin (AZT; all at 0.1, 0.3, 1, 3, 10, $30 \mu \mathrm{M}$ )) for 6 h. After fixation, GFP-LC3 dots were analyzed as a proxy for autophagy. Representative images are depicted in $\mathbf{A}(A Z T, C Q$, and $H C Q, 30 \mu M)$ and normalized data are shown as bar chart in $\mathbf{B}$. Data are means \pm SD of four replicates ${ }^{* *} P<0.01,{ }^{* * *} P<0.001$ vs. WT; Student's $t$-test). C, D U2OS WT or PelF2a S51A knockin cells both

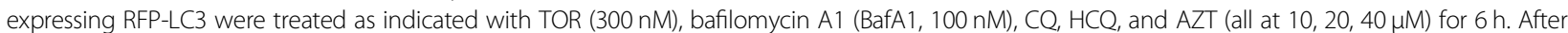
fixation, RFP-LC3 dots were analyzed by fluorescent microscopy. Representative images are shown in C (AZT, CQ, and HCQ, $40 \mu M)$ and normalized data are quantitated as a bar plot in $\mathbf{D}$. Data are means \pm SD of four replicates $\left({ }^{* *} P<0.01,{ }^{* *} P<0.001\right.$ vs. WT; Student's $t$-test). Scale bars equal $10 \mu m$. 


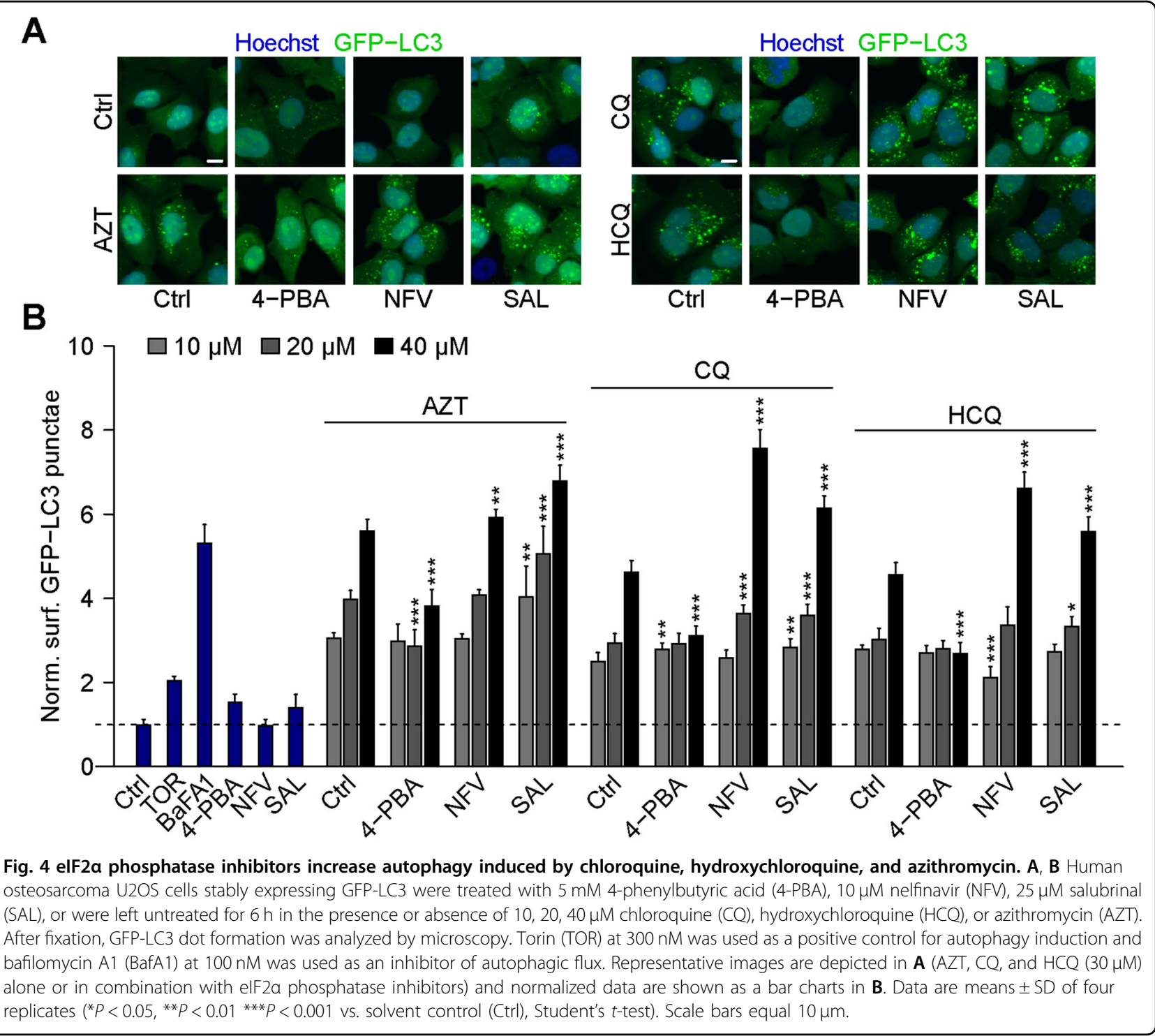

interaction $^{27}$. Hence, the question comes up whether the reported in vitro antiviral effects of $C Q$ and $\mathrm{HCQ}^{27,60}$ are linked to their capacity to elicit the ISR, thus augmenting an innate immune response (such as the initiation of a type-1 interferon response $e^{61,62}$, beyond their action on acidophilic cellular compartments ${ }^{63-65}$.

The ISR also has a fundamental role in ICD. In a plausible scenario, cells infected by viruses ultimately succumb to viral infection. If the virus (or other agents) induce the ISR, cell death would be perceived as immunogenic, hence favoring the stimulation of an immune response that involves dendritic cells as antigen presenters that then "educate" cytotoxic $\mathrm{T}$ lymphocytes to recognize MHC class I-restricted viral peptides expressed on the surface of infected cells ${ }^{37,66}$. By clearing infected cells, the immune system then can remove all virus-replicative niches from the body to subsequently establish a memory response that protects the patient from challenges by the same or antigenically similar viruses.

We have found in the past that artificial induction of the ISR by agents that stimulate an ER stress response (such as thapsigargin injected into tumors) or inhibit the dephosphorylation of eIF2 $\alpha$ (such as salubrinal and a peptides inhibiting the phosphatase PP1 interacting with its cofactor GADD34) can vigorously stimulate anticancer immune responses linked to $\mathrm{ICD}^{67-70}$. In this context, it is noteworthy that agents that selectively stimulate ISR but not any other manifestation of the unfolded stress response (such as the activation of ATF6 and that of IRE1/XBP1) are more efficient ICD inducers than agents with a broad effect on several arms of the unfolded stress response ${ }^{33,71,72}$. In quantitative terms, when compared to appropriate positive controls (thapsigargin, tunicamycin), AZT, CQ, and HCQ induced a strong ISR but scarce ATF6 and IRE1/XBP1 activation. Hence, 


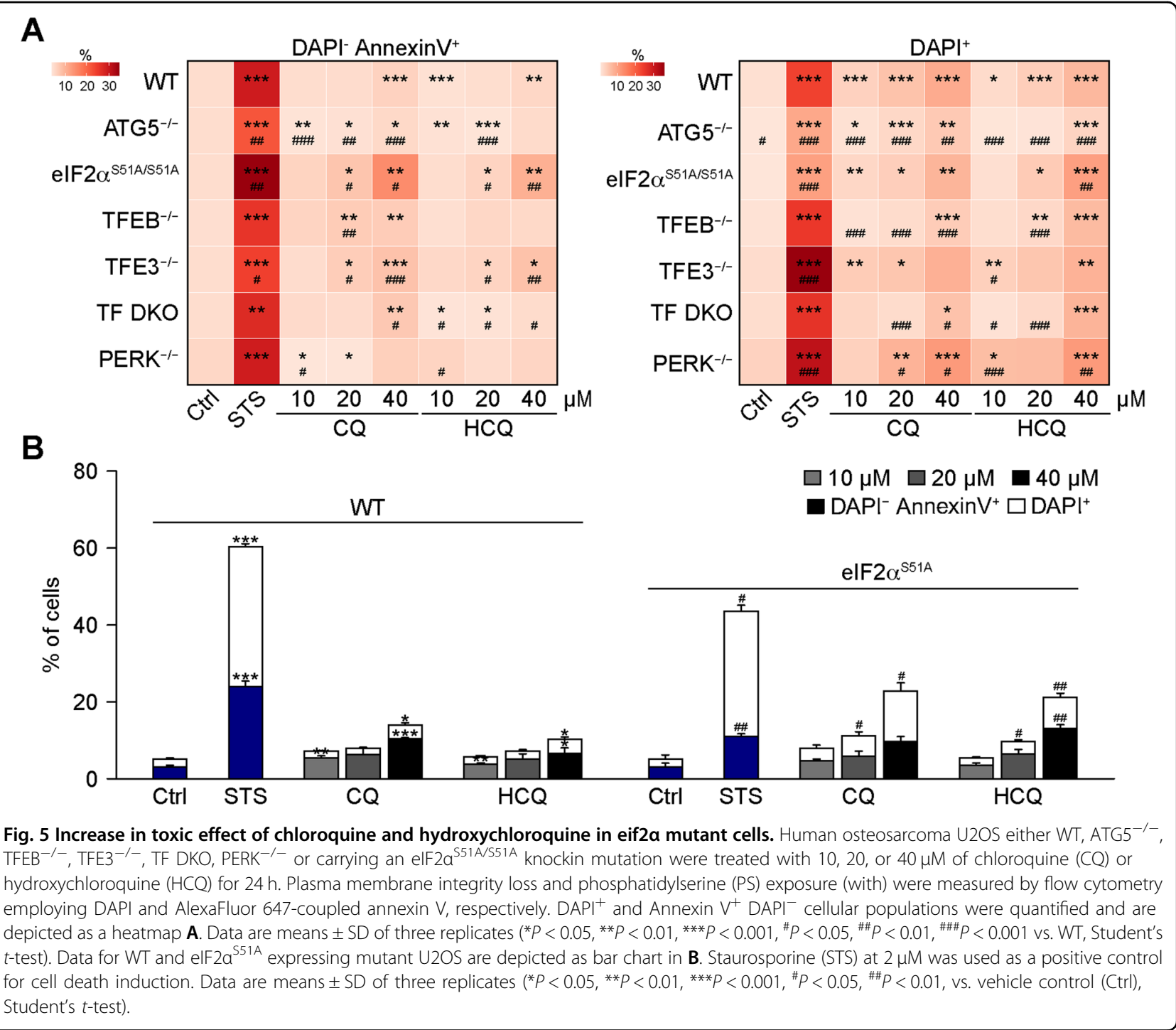

these lysosomotropic agents induce a pattern of response that would be compatible with a pro-ICD action. However, further virological and immunological experimentation will be required to (in)validate this conjecture.

In essence, our results demonstrate that AZT, CQ, and HCQ stimulate the ISR. This might contribute to the potential antiviral and immunostimulatory effects of such lysosomotropic agents. However, to definitively prove the mechanistic relevance of such effects, it would be necessary to develop small animal models ${ }^{73}$ in which AZT, CQ, and $\mathrm{HCQ}$, alone or in combination would have significant and reproducible antiviral activity.

\section{Materials and methods}

\section{Cell culture and chemicals}

Culture media and supplements for cell culture were purchased from Gibco-Life Technologies (Carlsbad, CA, USA) and plastic ware came from Greiner Bio-One
(Kremsmünster, Austria) and Corning (Corning, NY, USA). Wild-type human osteosarcoma U2OS or human glioma $\mathrm{H} 4$ cells were purchased from the American Type Culture Collection (ATCC, Rockefeller, MD, USA), their derivatives stably expressing GFP-LC3, RFP-LC3, or RFPGFP-LC3 were cultured in Dulbecco's modified Eagle's medium (DMEM) supplemented with $10 \%(\mathrm{v} / \mathrm{v})$ fetal bovine serum (FBS), $10 \mathrm{U} \mathrm{mL}^{-1}$ penicillin sodium, and $10 \mu \mathrm{g} \mathrm{mL}^{-1}$ streptomycin sulfate at $37^{\circ} \mathrm{C}$ in a humidified atmosphere with $5 \% \mathrm{CO}_{2}$. TFEB-deficient $\left(\mathrm{TFEB}^{-1-}\right.$ ), TFE3-deficient (TFE3 ${ }^{-1-}$ ), TFEB and TFE3-double deficient (TF DKO), ATG5-deficient $\left(\mathrm{ATG}^{-1-}\right.$ ), and PERKdeficient $\left(\mathrm{PERK}^{-1-}\right) \mathrm{U} 2 \mathrm{OS}$ cells were generated by means of CRISPR/Cas9-mediated genome editing, as per the manufacturer's recommendations ${ }^{31,74}$. U2OS cells stably expressing RFP-LC3 bearing a non-phosphorylatable mutant of eIF $2 \alpha\left(\right.$ eIF $\left.2 \alpha^{\mathrm{S} 51 \mathrm{~A}}\right)$ were constructed by means of CRISPR/Cas9 knockin as previously detailed ${ }^{31}$. In 

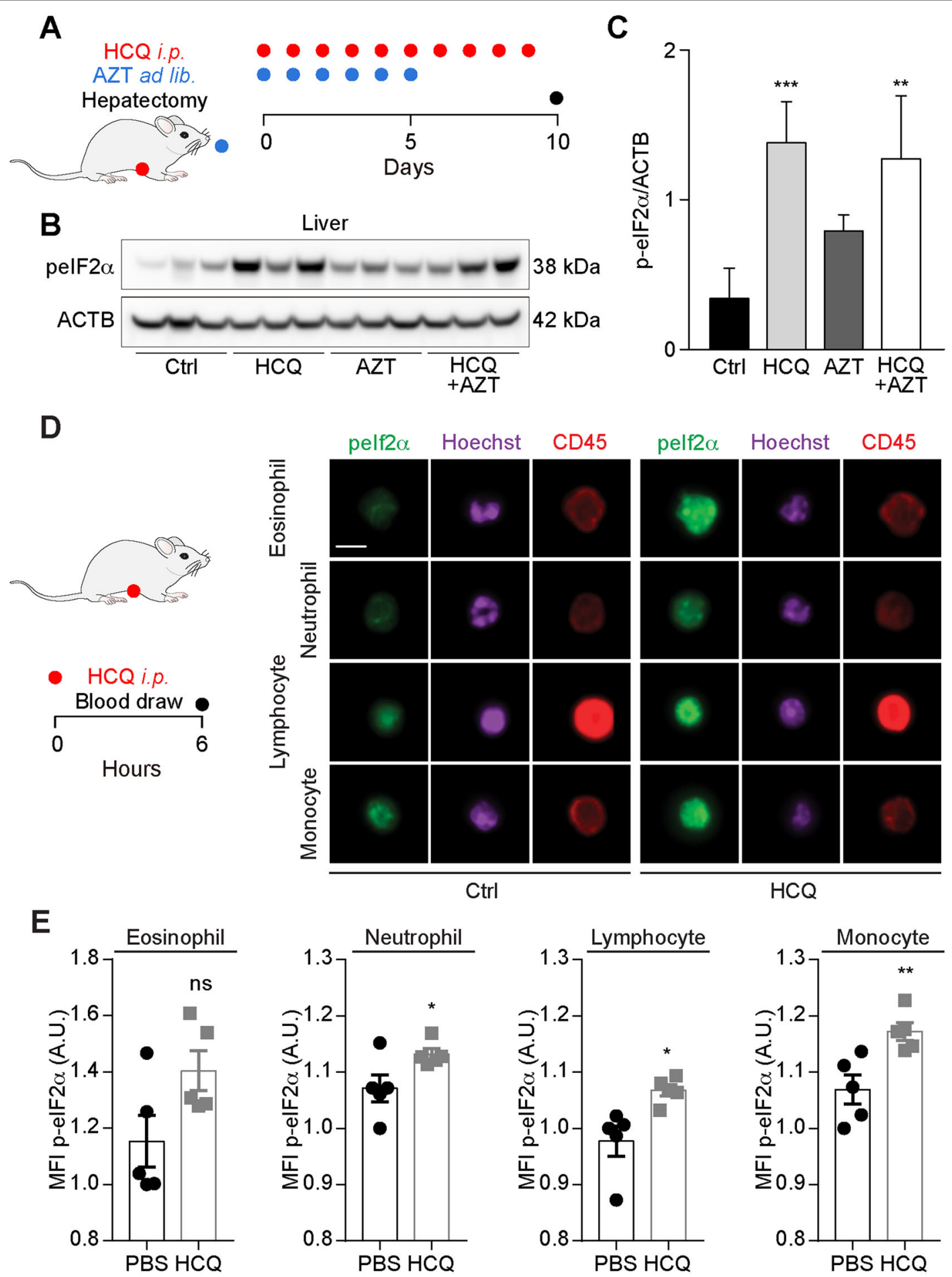

Fig. 6 Effects of hydroxychloroquine plus azithromycin in vivo. Mice were treated intraperitoneally (i.p.) with $50 \mathrm{mg} / \mathrm{kg} /$ day hydroxychloroquine (HCQ), orally fed with azithromycin (AZT) (3 mg/L in drinking water), or their combination as illustrated in the scheme (A). Livers were excised from three mice by group at the end of the treatment and the tissues were subjected to protein extraction for SDS-PAGE and immunoblot to detect the phosphorylation of pelF2a (B). $\beta$-Actin (ACTB) was used as a loading control. Band intensities were quantified by densitometry and the ratio of pelF2a to ACTB was calculated. Data are expressed as means \pm SEM of three mice $(\mathbf{C})$. Statistical significance is indicated as ${ }^{* *} P<0.01$ and ${ }^{* * *} P<0.001$ as compared with untreated control (Ctrl) (Student's t-test). D, E Mice were treated with $50 \mathrm{mg} / \mathrm{kg} \mathrm{HCQ}$ i.p. and blood was collected after $6 \mathrm{~h}$ to determine the level of pelF2a by immunofluorescence and image flow cytometry in the depicted leukocyte populations. Representative images are shown in $\mathbf{D}$. The scale bar equals $10 \mu \mathrm{m}$. Data are expressed as mean fluorescens intensities (MFI) means \pm SEM of five mice (E). Statistical comparisons were done by two-tailed unpaired Student's $t$-test $(\mathbf{E})$ comparing HCQ-treated to control mice that received PBS $\left({ }^{*} P<0.05,{ }^{* *} P<0.01\right)$. 
addition, U2OS cells stably expressing GFP-TFEB, a GFP under the DDIT3 promoter (CHOP::GFP), GFP-ATF6, and XBP1s- $\triangle \mathrm{DBD}$-venus were generated by our group in the past ${ }^{33,74}$. Chloroquine diphosphate salt (CQ, \#C6628), hydroxychloroquine sulfate (HCQ, \#PHR1782), azithromycin (AZT, \#75199), 4-phenylbutyric acid (4-PBA, \#P21005), nelfinavir (NFV, \#CDS021783), salubrinal (SAL, \#324895), thapsigargin (TG, \#T9033), tunicamycin (TM, \#T7765), bafilomycin A1 (BafA1, \#B1793), tumor necrosis factor- $\alpha$ (TNF- $\alpha$, \#T6674), torin1 (TOR, \#475991), and staurosporine (STS, \#S4400) were purchased from Sigma-Aldrich (St. Louis, MO, USA).

\section{High-content microscopy}

Human osteosarcoma U2OS-GFP-LC3 wild type or TFEB and TFE3-double deficient (TF DKO), ATG5 deficient $\left(\mathrm{ATG}^{-1-}\right.$ ), RFP-GFP-LC3, RFP-LC3 wild type or mutant cells expressing a non-phosphorylatable knockin of eIF $2 \alpha\left(\right.$ eIF $\left.2 \alpha^{\text {S51A/S51A }}\right)$ were seeded in 384well $\mu$ clear imaging plates (Greiner Bio-One) at a density of $2 \times 10^{3}$ cells per well and allowed to adapt for overnight. Furthermore, ATG5 ${ }^{-1-}$, eIF $2 \alpha^{\mathrm{S} 51 \mathrm{~A} / \mathrm{S} 51 \mathrm{~A}}$, and TF DKO cells were treated for $6 \mathrm{~h}$. Moreover, $2 \times 10^{3}$ U2OS cells either wild type or stably expressing GFPATF6, CHOP::GFP, GFP-TFEB, or XBP1- $\triangle$ DBD-venus were seeded in 384-well black imaging plates (Greiner Bio-One) and let adhere overnight. Cells were then treated for $6 \mathrm{~h}$ to assess TFEB translocation, and $24 \mathrm{~h}$ to monitor abundance of ATF6 and spliced XBP1 (XBP1s), or to measure CHOP expression. Next, cells were fixed with $3.7 \%$ formaldehyde (\#F8775; SigmaAldrich) supplemented with $1 \mu \mathrm{g} / \mathrm{ml}$ Hoechst 33342 (\#H3570; Thermo Fisher Scientific) at $4{ }^{\circ} \mathrm{C}$ overnight. After washing the cells, the plates were sealed and analyzed by automated microscopy. Image acquisition was performed using an ImageXpress Micro XL automated microscope (Molecular Devices, Sunnyvale, CA, US) equipped with a $\times 20$ PlanApo objective (Nikon, Tokyo, Japan), followed by automated image segmentation. A minimum of four images were acquired per well, and experiments involved at least triplicate assessments.

\section{Image segmentation and data analysis}

Upon acquisition, images were segmented and analyzed using R. Briefly, cells were segmented and divided into nuclear and cytoplasmic regions based on the nuclear Hoechst staining and cytoplasmic GFP or RFP signal. After exclusion of cellular debris and dead cells, parameters of interest were normalized, statistically evaluated, and graphically depicted with R software. Using R, images were extracted and pixel intensities scaled to be visible (in the same extent for all images of a given experiment).

\section{Immunofluorescence}

Cells were treated for $16 \mathrm{~h}$ to detect eIF $2 \alpha$ phosphorylation (PeIF2 $\alpha$ ) and TFE3 expression, or $6 \mathrm{~h}$ to measure p65 nuclear translocation. Then cells were fixed with $3.7 \%$ PFA at $4{ }^{\circ} \mathrm{C}$ overnight. For the immunostaining of TFE3, p65, and phospho-eIF2 $\alpha$ (Ser51), fixed cells were permeabilized with $0.1 \%$ Triton X-100 (\#X100; SigmaAldrich) on ice, and unspecific antibody binding was blocked with $5 \%$ bovine serum albumin (BSA, w/v in PBS) for $1 \mathrm{~h}$. Then cells were incubated with antibodies specific to TFE3 (\#ab93808, 1:200; Abcam), phospho-eIF2 alpha (Ser51) (\#ab32157, 1:1000; Abcam), or p65 (\#4764, 1:100; Cell Signalling Technology) at $4{ }^{\circ} \mathrm{C}$ overnight. After washing with PBS twice, AlexaFluor 568-conjugated secondary antibodies (Thermo Fisher Scientific) were employed for additional $2 \mathrm{~h}$ at RT. Then cells were washed and imaged by automated fluorescence microscopy as described above. The nuclear-to-cytoplasm intensity ratio of TFE3 and p65 as well as the cytoplasmic intensity of phospho-eIF2 $\alpha$ (Ser51) were measured and normalized to controls.

\section{Imaging cytofluorometric analysis}

Six hundred microliters of total blood were diluted in $25 \mathrm{~mL}$ red blood cell lysis buffer (BioLegend) and incubated for $10 \mathrm{~min}$ at room temperature. Then the cells were washed twice in PBS, fixed with 4\% PFA for $20 \mathrm{~min}$ at room temperature, permeabilized with $0.25 \%$ Tween-20 for 15 min at $4{ }^{\circ} \mathrm{C}$, and blocked with $2 \%$ BSA in PBS. Cells were incubated with anti-phospho-eIF2 alpha (Ser51) and AlexaFluor 647-conjugated anti-mouse PTPRC/CD45 antibody (\#clone 30-F11; BioLegend) for $1 \mathrm{~h}$ at room temperature. Then cells were incubated for $1 \mathrm{~h}$ with donkey AlexaFluor488-conjugated secondary antibody and Hoechst $33342(0.5 \mu \mathrm{g} / \mu \mathrm{L})$. Multispectral imaging flow cytometry was performed on an AMNIS ImageStream X Mark II equipped with 375-, 488-, 561-, and $642 \mathrm{~nm}$ lasers using the $\times 60$ magnification lens. At least 6000 cells/sample were acquired for each sample. The analysis was performed with IDEAS software v6.2. Exclusively focused images were included in the analysis. Selection was based on the gradient RMS feature of bright field images. A compensation matrix was calculated using single color fluorescent controls. This matrix was applied to each file and singlets were then gated on aspect ratio vs. area of bright field and leukocyte subpopulations were gated on a pictogram indicating the intensity of PTPRC/ CD45 staining vs. dark field. Following the intensity of peIF2 $\alpha$ was quantified in each cell.

\section{Quantification of cell death by flow cytometry}

Cell death was assessed by means of the Alexa Fluor 647 Annexin V (\#640943; BioLegend) and DAPI (\#62248; Thermo Fisher Scientific) kit following the manufacturer's 
instructions. Briefly, cells were seeded in 12-well plates (with $5 \times 10^{4}$ cells per well) and incubated at $37^{\circ} \mathrm{C}$ in a humidified atmosphere with $5 \% \mathrm{CO}_{2}$ for $24 \mathrm{~h}$, then cells were collected and washed in PBS containing 0.5\% BSA before the cell pellet was resuspended in $100 \mu \mathrm{L}$ of Annexin V Binding Buffer (\#422201, BioLegend) containing Alexa Fluor 647-coupled Annexin V. Samples were then incubated at room temperature in the dark for $15 \mathrm{~min}$ before adding $100 \mu \mathrm{L}$ of PBS containing $0.5 \%$ BSA and $2 \mu \mathrm{g} / \mathrm{mL}$ DAPI solution. Acquisitions were performed on a BD LSRFortessa ${ }^{\mathrm{TM}}$ cell analyzer (BD Biosciences, San Jose, California, USA), and data were statistically analyzed using the FlowJo 10.5.3 software (Tree Star, Ashland, Oregon, USA).

\section{In vivo experimentation}

The animal experiment was approved by the Gustave Roussy ethics committee with the project number: 24771-2020032413235413, and all procedures were performed in compliance with the governmental and institutional guidelines and regulations. Mice were kept in a temperature-controlled SPF environment (12 h light/dark cycles) with food and water ad libitum. Eight-week-old female $\mathrm{C} 57 \mathrm{Bl} / 6 \mathrm{j}$ mice were obtained from ENVIGO (France). To quantify the in vivo phosphorylation eIF2 $\alpha$ (S51), naive mice were intraperitoneally (i.p.) treated with HCQ at a dose of $50 \mathrm{mg} / \mathrm{kg} /$ day in $200 \mu \mathrm{L}$ PBS daily for 10 days $^{75,76}$; fed with AZT in autoclaved drinking water at a concentration of $3 \mathrm{mg} / \mathrm{L}$ (purchased from the local pharmacy) for 5 days, and the solution was changed daily throughout the treatment period ${ }^{77,78}$. All mice were sacrificed at day $10,4 \mathrm{~h}$ post-injection with $\mathrm{HCQ}$, and livers and hearts were snap frozen in liquid nitrogen.

\section{Immunoblotting}

Thirty milligrams of liver tissue were dissociated in Precellys lysing tubes (\#CK28; Bertin Technologies SAS, France) containing $1 \mathrm{~mL}$ of RIPA lysis buffer (\#89901; Invitrogen) by using the Precellys 24 homogenizer (Bertin Technologies SAS) at 6500 r.p.m. for $60 \mathrm{~s}$, followed by spinning at $14 \mathrm{~s} 10^{3} \mathrm{~d} g$ for 15 min to collect the supernatant that contains soluble proteins. Protein concentration was measured by means of by the BCA Assay (Bio-Rad, Hercules, CA, USA). The protein solution was mixed with $4 \times$ loading buffer (\#NP0008; Invitrogen), and denatured at $100^{\circ} \mathrm{C}$ for $10 \mathrm{~min}$ before subjected to western blotting. Forty micrograms of total protein were resolved on $4-12 \%$ NuPAGE Bis-Tris protein gels (\#NP0336BOX; Invitrogen) and transferred to PVDF membranes (\#IPFL00010; Merck Millipore). The membranes were blocked with $5 \%$ non-fat dry milk in TBST for $1 \mathrm{~h}$ before incubating with primary antibodies to phospho-elF2 alpha (Ser51) (\#ab32157, 1:1000; Abcam) overnight at $4{ }^{\circ} \mathrm{C}$. Membranes were washed several times with TBST for $10 \mathrm{~min}$ each before incubation with HRP-conjugated secondary antibody (\#4050-05;
SouthernBiotech) for $2 \mathrm{~h}$ at room temperature. At last, the membranes were washed again and subjected to chemiluminescence detection with the Amersham ECL Prime detection reagent kit (\#RPN2236; GE Healthcare) on an ImageQuant LAS 4000 software-assisted imager. The exposed membranes were stripped and re-probed with antibodies specific to $\beta$-actin (\#ab20727; Abcam) as loading control using the procedure described above. Densitometry was performed using the ImageQuant TL software (GE Healthcare, Piscataway, NJ, USA).

\section{Image and data processing}

Images were segmented using the EBImage package (available from Bioconductor repository https://www. bioconductor.org) with the $\mathrm{R}$ software. The nuclear region was defined using a polygon mask based on the nuclear Hoechst signal, and a second polygon mask was generated using the cytoplasmic GFP or RFP signal. For the assessment of autophagic vesicles, a third mask was created on cytoplasmic regions exhibiting a high intensity signal of GFP or RFP corresponding with LC3 aggregates.

Following image segmentation, the data were extracted and reduced using the $\mathrm{R}$ software. After exclusion of cellular debris and dead cells, parameters of interest were normalized to controls, statistically evaluated, and graphically depicted with $\mathrm{R}$ software. Using $\mathrm{R}$, images were extracted and pixel intensities scaled to be visible (to the same extent for all images of a given experiment).

\section{Statistical analysis}

Unless otherwise mentioned, data are reported as means \pm SD of triplicate determinations and experiments were repeated at least three times yielding similar results, and statistical significance was assessed by Student's $t$-test with a $P$ value adjustment based on the Benjamini-Hochberg procedure.

\section{Acknowledgements}

This study contributes to the IdEx Université de Paris ANR-18-IDEX-0001. A.-L.T. and Q.W. are supported by a CSC fellowship. G.K. is supported by the Ligue contre le Cancer (équipe labellisée); Agence National de la Recherche (ANR) Projets blancs; ANR under the frame of E-Rare-2, the ERA-Net for Research on Rare Diseases; AMMICa US23/CNRS UMS3655; Association pour la recherche sur le cancer (ARC); Association "Ruban Rose"; Cancéropôle Ile-de-France;

Chancelerie des universités de Paris (Legs Poix), Fondation pour la Recherche Médicale (FRM); a donation by Elior; European Research Area Network on

Cardiovascular Diseases (ERA-CVD, MINOTAUR); Gustave Roussy Odyssea, the European Union Horizon 2020 Project Oncobiome; Fondation Carrefour; Highend Foreign Expert Program in China (GDW20171100085), Institut National du Cancer (INCa); Inserm (HTE); Institut Universitaire de France; LeDucq Foundation; the LabEx Immuno-Oncology (ANR-18-IDEX-0001); the RHU Torino Lumière; the Seerave Foundation; the SIRIC Stratified Oncology Cell DNA Repair and Tumor Immune Elimination (SOCRATE); and the SIRIC Cancer Research and Personalized Medicine (CARPEM).

\footnotetext{
Author details

${ }^{1}$ Centre de Recherche des Cordeliers, Equipe labellisée par la Ligue contre le cancer, Université de Paris, Sorbonne Université, Inserm U1138, Institut Universitaire de France, Paris, France. ${ }^{2}$ Metabolomics and Cell Biology Platforms, Gustave Roussy, Villejuif, France. ${ }^{3}$ Department of Breast and Thyroid Surgery, Renmin Hospital of Wuhan University, Wuhan, Hubei, P. R. China.
} 
${ }^{4}$ Suzhou Institute for Systems Medicine, Chinese Academy of Medical Sciences, Suzhou, China. ${ }^{5}$ Pôle de Biologie, Hôpital Européen Georges Pompidou, AP-HP, Paris, France. 'Karolinska Institutet, Department of Women's and Children's Health, Karolinska University Hospital, Stockholm, Sweden

\section{Author contributions}

Concept and design: O.K. and G.K. Writing, review, and/or revision of the manuscript: O.K., M.L., and G.K. Experimentation and analysis: A.-L.T., Q.W., P.L., L.Z., I.M., and M.L. All authors approve the final version of the article.

\section{Conflict of interest}

G.K. and O.K. are cofounders of Samsara Therapeutics, a biotech company developing autophagy inducers for the treatment of aging. The other authors have no relevant conflict of interest to declare.

\section{Ethics approval}

The animal experiment was approved by the Gustave Roussy ethics committee with the project number: 24771-2020032413235413, and all procedures were performed in compliance with the governmental and institutional guidelines and regulations.

\section{Publisher's note}

Springer Nature remains neutral with regard to jurisdictional claims in published maps and institutional affiliations.

Supplementary Information accompanies this paper at (https://doi.org/ 10.1038/s41419-020-03324-w).

Received: 4 August 2020 Revised: 1 December 2020 Accepted: 4 December 2020

Published online: 06 January 2021

\section{References}

1. Gautret, P. et al. Hydroxychloroquine and azithromycin as a treatment of COVID-19: results of an open-label non-randomized clinical trial. Int. J. Antimicrob. Agents 56, 105949 (2020).

2. Holopigian, K., Snow, J., Seiple, W. \& Siegel, I. Variability of the pattern electroretinogram. Doc. Ohthalmol. 70, 103-115 (1988).

3. Gautret, P. et al. Clinical and microbiological effect of a combination of hydroxychloroquine and azithromycin in 80 COVID-19 patients with at least a six-day follow up: A pilot observational study. Travel Med. Infect. Dis. 34, 101663 (2020).

4. Lagier, J. C. et al. Outcomes of 3,737 COVID-19 patients treated with hydroxychloroquine/azithromycin and other regimens in Marseille, France: a retrospective analysis. Travel Med. Infect. Dis. 101791, https://doi.org/10.1016/j. tmaid.2020.101791 (2020).

5. Mikami, T. et al. Risk factors for mortality in patients with COVID-19 in New York City. J. Gen. Intern. Med. https://doi.org/10.1007/s11606-02005983-z (2020).

6. Tang, W. et al. Hydroxychloroquine in patients with mainly mild to moderate coronavirus disease 2019: open label, randomised controlled trial. BMJ 369, m1849 (2020)

7. Boulware, D. R. et al. A randomized trial of hydroxychloroquine as postexposure prophylaxis for Covid-19. N. Engl. J. Med. https://doi.org/10.1056/ NEJMoa2016638 (2020).

8. Das, S., Bhowmick, S., Tiwari, S. \& Sen, S. An updated systematic review of the therapeutic role of hydroxychloroquine in coronavirus disease-19 (COVID-19). Clin. Drug Investig. 40, 591-601 (2020).

9. Liu, W. et al. Efficacy and safety of antiviral treatment for COVID-19 from evidence in studies of SARS-COV-2 and other acute viral infections: a systematic review and meta-analysis. CMAJ 192, E734-E744 (2020).

10. Rainsford, K. D., Parke, A. L., Clifford-Rashotte, M. \& Kean, W. F. Therapy and pharmacological properties of hydroxychloroquine and chloroquine in treatment of systemic lupus erythematosus, rheumatoid arthritis and related diseases. Inflammopharmacology 23, 231-269 (2015).
11. Schrezenmeier, E. \& Dorner, T. Mechanisms of action of hydroxychloroquine and chloroquine: implications for rheumatology. Nat. Rev. Rheumatol. 16 155-166 (2020).

12. Weissmann, G. Labilization and stabilization of lysosomes. Fed. Proc. 23 1038-1044 (1964)

13. Ignarro, L. J. Effects of anti-inflammatory drugs on the stability of rat liver lysosomes in vitro. Biochem. Pharmacol. 20, 2847-2860 (1971).

14. Carevic, O. \& Djokic, S. Comparative studies on the effects of erythromycin A and azithromycin upon extracellular release of lysosomal enzymes in inflammatory processes. Agents Actions 25, 124-131 (1988).

15. Kuzu, O. F., Toprak, M., Noory, M. A. \& Robertson, G. P. Effect of lysosomotropic molecules on cellular homeostasis. Pharmacol. Res. 117, 177-184 (2017).

16. Pisonero-Vaquero, S. \& Medina, D. L. Lysosomotropic drugs: pharmacological tools to study lysosomal function. Curr. Drug Metab. 18, 1147-1158 (2017).

17. Ploper, D. \& De Robertis, E. M. The MITF family of transcription factors: role in endolysosomal biogenesis, Wnt signaling, and oncogenesis. Pharmacol. Res. 99, 36-43 (2015).

18. Wisner-Gebhart, A. M., Brabec, R. K. \& Gray, R. H. Morphometric studies of chloroquine-induced changes in hepatocytic organelles in the rat. Exp. Mol. Pathol. 33, 144-152 (1980).

19. Boya, P. et al. Inhibition of macroautophagy triggers apoptosis. Mol. Cell. Biol. 25, 1025-1040 (2005).

20. Renna, M. et al. Azithromycin blocks autophagy and may predispose cystic fibrosis patients to mycobacterial infection. J. Clin. Investig. 121, 3554-3563 (2011).

21. Boya, P. et al. Mitochondrial membrane permeabilization is a critical step of lysosome-initiated apoptosis induced by hydroxychloroquine. Oncogene $\mathbf{2 2}$, 3927-3936, (2003).

22. Manic, G., Obrist, F., Kroemer, G., Vitale, I. \& Galluzzi, L. Chloroquine and hydroxychloroquine for cancer therapy. Mol. Cell. Oncol. 1, e29911 (2014).

23. Kroemer, G. \& Galluzzi, L. Lysosome-targeting agents in cancer therapy. Oncotarget 8, 112168-112169 (2017).

24. Hetz, C., Zhang, K. \& Kaufman, R. J. Mechanisms, regulation and functions of the unfolded protein response. Nat. Rev. Mol. Cell Biol. 21, 421-438, (2020).

25. Kepp, O. et al. Viral subversion of immunogenic cell death. Cell Cycle 8 860-869, https://doi.org/10.4161/cc.8.6.7939 (2009).

26. Johnston, B. P. \& McCormick, C. Herpesviruses and the unfolded protein response. viruses 12, https://doi.org/10.3390/v12010017 (2019).

27. Rabouw, H. H. et al. Inhibition of the integrated stress response by viral proteins that block p-elF2-elF2B association. Nat. Microbiol. https://doi.org/ 10.1038/s41564-020-0759-0 (2020).

28. Talloczy, Z. et al. Regulation of starvation- and virus-induced autophagy by the elF2alpha kinase signaling pathway. Proc. Natl Acad. Sci. USA 99, 190-195 (2002).

29. Kroemer, G., Marino, G. \& Levine, B. Autophagy and the integrated stress response. Mol. Cell 40, 280-293 (2010).

30. Costa-Mattioli, M. \& Walter, P. The integrated stress response: from mechanism to disease. Science $\mathbf{3 6 8}$, https://doi.org/10.1126/science.aat5314 (2020).

31. Humeau, J. et al. Phosphorylation of eukaryotic initiation factor-2alpha (elF2alpha) in autophagy. Cell Death Dis. 11, 433 (2020).

32. Kepp, O. et al. elF2alpha phosphorylation as a biomarker of immunogenic cell death. Semin. Cancer Biol. 33, 86-92 (2015).

33. Bezu, L. et al. elF2alpha phosphorylation is pathognomonic for immunogenic cell death. Cell Death Differ. 25, 1375-1393 (2018).

34. Humeau, J. et al. Inhibition of transcription by dactinomycin reveals a new characteristic of immunogenic cell stress. EMBO Mol. Med. 12, e11622 (2020).

35. Uhl, M. et al. Autophagy within the antigen donor cell facilitates efficient antigen cross-priming of virus-specific CD8+ T cells. Cell Death Differ. 16, 991-1005 (2009).

36. Galluzzi, L., Buque, A., Kepp, O., Zitvogel, L. \& Kroemer, G. Reply: the complement system is also important in immunogenic cell death. Nat. Rev. Immunol. 17, 143 (2017).

37. Tappe, K. A. et al. Immunogenic cell death of dendritic cells following modified vaccinia virus Ankara infection enhances CD8(+) T cell proliferation. Eur. J. Immunol. 48, 2042-2054 (2018).

38. Martins, I. et al. Molecular mechanisms of ATP secretion during immunogenic cell death. Cell Death Differ. 21, 79-91 (2014).

39. Madeo, F. et al. Phylogenetic conservation of the preapoptotic calreticulin exposure pathway from yeast to mammals. Cell Cycle 8, 639-642 (2009). 
40. Senovilla, L., Demont, Y., Humeau, J., Bloy, N. \& Kroemer, G. Image cytofluorometry for the quantification of ploidy and endoplasmic reticulum stress in cancer cells. Methods Mol. Biol. 1524, 53-64 (2017).

41. Giglio, P. et al. PKR and GCN2 stress kinases promote an ER stress-independent elF2alpha phosphorylation responsible for calreticulin exposure in melanoma cells. Oncoimmunology 7, e1466765 (2018)

42. Bravo-San Pedro, J. M. et al. High-throughput quantification of GFP-LC3(+) dots by automated fluorescence microscopy. Methods Enzymol. 587, 71-86 (2017).

43. Klionsky, D. J. et al. Guidelines for the use and interpretation of assays for monitoring autophagy (3rd edition). Autophagy 12, 1-222 (2016).

44. Perera, R. M., Di Malta, C. \& Ballabio, A. MiT/TFE family of transcription factors, lysosomes, and cancer. Annu. Rev. Cancer Biol. 3, 203-222 (2019).

45. Bezu, L., Wu Chuang, A., Humeau, J., Kroemer, G. \& Kepp, O. Quantification of elF2alpha phosphorylation during immunogenic cell death. Methods Enzymol. 629, 53-69 (2019)

46. Saftig, P. \& Haas, A. Turn up the lysosome. Nat. Cell Biol. 18, 1025-1027, https:// doi.org/10.1038/ncb3409 (2016).

47. Di Malta, C., Cinque, L. \& Settembre, C. Transcriptional regulation of autophagy: mechanisms and diseases. Front. Cell Dev. Biol. 7, 114 (2019).

48. Acevo-Rodriguez, P. S., Maldonado, G., Castro-Obregon, S. \& Hernandez, G. Autophagy regulation by the translation machinery and its implications in cancer. Front. Oncol. 10, 322 (2020).

49. Kolb, P. S. et al. The therapeutic effects of 4-phenylbutyric acid in maintaining proteostasis. Int. J. Biochem. Cell Biol. 61, 45-52 (2015).

50. De Gassart, A. et al. An inhibitor of HIV-1 protease modulates constitutive elF2alpha dephosphorylation to trigger a specific integrated stress response. Proc. Natl Acad. Sci. USA 113, E117-E126 (2016).

51. Boyce, M. et al. A selective inhibitor of elF2alpha dephosphorylation protects cells from ER stress. Science 307, 935-939 (2005).

52. Pozarowski, P., Grabarek, J. \& Darzynkiewicz, Z. Current Protocols in Cell Biology Chapter 18, 18.8.1-18.8.33 (John Wiley \& Sons, Inc., 2004).

53. Ruiz, A. et al. Effect of hydroxychloroquine and characterization of autophagy in a mouse model of endometriosis. Cell Death Dis. 7, e2059 (2016).

54. Burikhanov, R. et al. Chloroquine-inducible Par-4 secretion is essential for tumor cell apoptosis and inhibition of metastasis. Cell Rep. 18, 508-519 (2017).

55. Collins, K. P., Jackson, K. M. \& Gustafson, D. L. Hydroxychloroquine: a physiologically-based pharmacokinetic model in the context of cancer-related autophagy modulation. J. Pharmacol. Exp. Therap. $\mathbf{3 6 5}$ 447-459 (2018).

56. Qiao, X. et al. Hydroxychloroquine Improves obesity-associated insulin resistance and hepatic steatosis by regulating lipid metabolism. Front. Pharmacol. 10, 855 (2019).

57. Florey, O., Gammoh, N., Kim, S. E., Jiang, X. \& Overholtzer, M. V-ATPase and osmotic imbalances activate endolysosomal LC3 lipidation. Autophagy 11, 88-99 (2015).

58. Jheng, J. R., Ho, J. Y. \& Horng, J. T. ER stress, autophagy, and RNA viruses. Front. Microbiol. 5, 388 (2014)

59. Liu, Y. et al. The role of host elF2alpha in viral infection. Virol. J. 17, 112 (2020).
60. Andreani, J. et al. In vitro testing of combined hydroxychloroquine and azithromycin on SARS-CoV-2 shows synergistic effect. Microb. Pathog. 145 104228 (2020).

61. Balachandran, S. et al. Essential role for the dsRNA-dependent protein kinase PKR in innate immunity to viral infection. Immunity 13, 129-141 (2000).

62. Liang, Q., Deng, H., Sun, C. W., Townes, T. M. \& Zhu, F. Negative regulation of IRF7 activation by activating transcription factor 4 suggests a cross-regulation between the IFN responses and the cellular integrated stress responses. J. Immunol. 186, 1001-1010 (2011).

63. Carmona-Gutierrez, D. et al. Digesting the crisis: autophagy and coronaviruses. Microb. Cell 7, 119-128 (2020).

64. Bonam, S. R., Muller, S., Bayry, J. \& Klionsky, D. J. Autophagy as an emerging target for COVID-19: lessons from an old friend, chloroquine. Autophagy https://doi.org/10.1080/15548627.2020.1779467 (2020).

65. Bello-Perez, M., Sola, I., Novoa, B., Klionsky, D. J. \& Falco, A. Canonical and noncanonical autophagy as potential targets for COVID-19. Cells https:/doi. org/10.3390/cells9071619 (2020).

66. Galluzzi, L., Buque, A., Kepp, O., Zitvogel, L. \& Kroemer, G. Immunogenic cell death in cancer and infectious disease. Nat. Rev. Immunol. 17, 97-111 (2017)

67. Obeid, M. et al. Ecto-calreticulin in immunogenic chemotherapy. Immunol. Rev. 220, 22-34 (2007).

68. Panaretakis, T. et al. Mechanisms of pre-apoptotic calreticulin exposure in immunogenic cell death. EMBO J. 28, 578-590 (2009).

69. Kepp, O. et al. Disruption of the PP1/GADD34 complex induces calreticulin exposure. Cell cycle 8, 3971-3977 (2009).

70. Martins, I. et al. Restoration of the immunogenicity of cisplatin-induced cancer cell death by endoplasmic reticulum stress. Oncogene 30, 1147-1158 (2011).

71. Pozzi, $C$. et al. The EGFR-specific antibody cetuximab combined with chemotherapy triggers immunogenic cell death. Nat. Med. 22, 624-631 (2016).

72. Liu, P. et al. Crizotinib-induced immunogenic cell death in non-small cell lung cancer. Nat. Commun. 10, 1486 (2019).

73. Park, S. J. et al. Antiviral efficacies of FDA-approved drugs against SARS-CoV-2 infection in ferrets. mBio https://doi.org/10.1128/mBio.01114-20 (2020).

74. Chen, G. et al. 3,4-Dimethoxychalcone induces autophagy through activation of the transcription factors TFE3 and TFEB. EMBO Mol. Med. https://doi.org/ 10.15252/emmm.201910469 (2019).

75. Rosenfeldt, M. T. et al. p53 status determines the role of autophagy in pancreatic tumour development. Nature 504, 296-300 (2013).

76. Vera-Ramirez, L., Vodnala, S. K., Nini, R., Hunter, K. W. \& Green, J. E. Autophagy promotes the survival of dormant breast cancer cells and metastatic tumour recurrence. Nat. Commun. 9, 1944 (2018).

77. Radhakrishnan, S. V. et al. Preventive azithromycin treatment reduces noninfectious lung injury and acute graft-versus-host disease in a murine model of allogeneic hematopoietic cell transplantation. Biol. Blood Marrow Transpl. 21, 30-38 (2015).

78. Liu, P. et al. Combination treatments with hydroxychloroquine and azithromycin are compatible with the therapeutic induction of anticancer immune responses. Oncoimmunology 9, 1789284 (2020). 